\section{Photoshop $^{\mathrm{TM}}$ Tutorials Selecting ROIs from Brightfield Images}

Jerry Sedgewick

University of Minnesota

sedge001@umn.edu

This column is, perhaps, a departure from many techniques papers because the accompanying image chosen as "representative" really is representative, rather than what is seen in many published papers in which the representative image is the one perfect image among hundreds of those that are biologically messy. The image used in this article to illustrate methods is not the best to use when creating regions of interest with Photoshop tools, for it contains elements which make the separation into regions of interest difficult to accomplish without reacquiring the image using better microscope techniques. I'm assuming that an image of this sort CAN be reacquired: if that isn't possible, some head-scratching and, perhaps a shift from a position of absolute accuracy to statistical accuracy might occur, and many would argue, should occur (since the whole matter is statistical anyway). Hopefully the investigator understands that, in some instances, human error can be as confounding as image processing error, except that image processing can create consistency. In other instances, artifacts in the images themselves can cause too many wrong features to be measured, leaving humans as the best instrument.

Having said that, the use of Photoshop to automatically or semiautomatically find regions of interest is always worth a try. The best results are spawned when careful attention is paid to the acquisition of images on the microscope. Here is a listing of what reduces headaches down the line:

1. Use Flatfield correction (also called "Shading correction," "Blank Field," etc.) in camera software when collecting images.

2. White Balance the camera if it is a color camera.

3. De-Interlace images if the camera is a video/analogue camera.

4. Avoid taking pictures in which white and black values are saturated (whites shouldn't be too white, blacks too black).

5. Adjust lighting on the microscope to achieve Kohler illumination (see http://micro.magnet.fsu.edu/primer/anatomy/kohler for more info).

6. Choose magnification on the microscope so that the lowest magnification is used at which features are positively identifiable.

7. Attempt to obtain images from as many sections as possible during a single session; and save a reference image for contrast, color and brightness matching for subsequent sessions.

Point \#5-the focusing of light-can be crucial to obtaining images in which regions of interest will be selected in Photoshop. The idea is to create as little diffraction as possible when light travels around features on the microscope. This is best accomplished either by opening the substage condenser's aperture to cover $10 \%$ of the field (Kohler illumination), or by opening the condenser all the way or by taking it off completely (and violating the rules of Kohler illumination). The image will look slightly unfocused, but the background features will be less likely to create unwanted surface textures that may be dark enough to be included with regions of interest.

Here are some steps to follow in Photoshop after having acquired these images:

Duplicate the image. Don't save over your original. Under Image on the menu, choose Duplicate.

Median Filter. Once these steps are taken, the joy of segmenting (selecting regions of interest) can begin. Nearly every image requires the use of the Median filter (under Filter select Noise, then Median) to blur the image while maintaining the borders of objects. Noise is easily seen by zooming in to areas containing the same tone (as in flat background areas) and then looking at differences in pixel darkness/lightness. Noisy images contain large differences and these are "flattened" by using the median filter. Most grayscale images require a small amount of filtering, and so the radius slider is set to a value of 1 . Color images, on the other hand, may need a higher value for radius in order to separate out colors more effectively. This is set visually: change the radius while looking at the image until colors "pool" together into what looks like a topographic map.

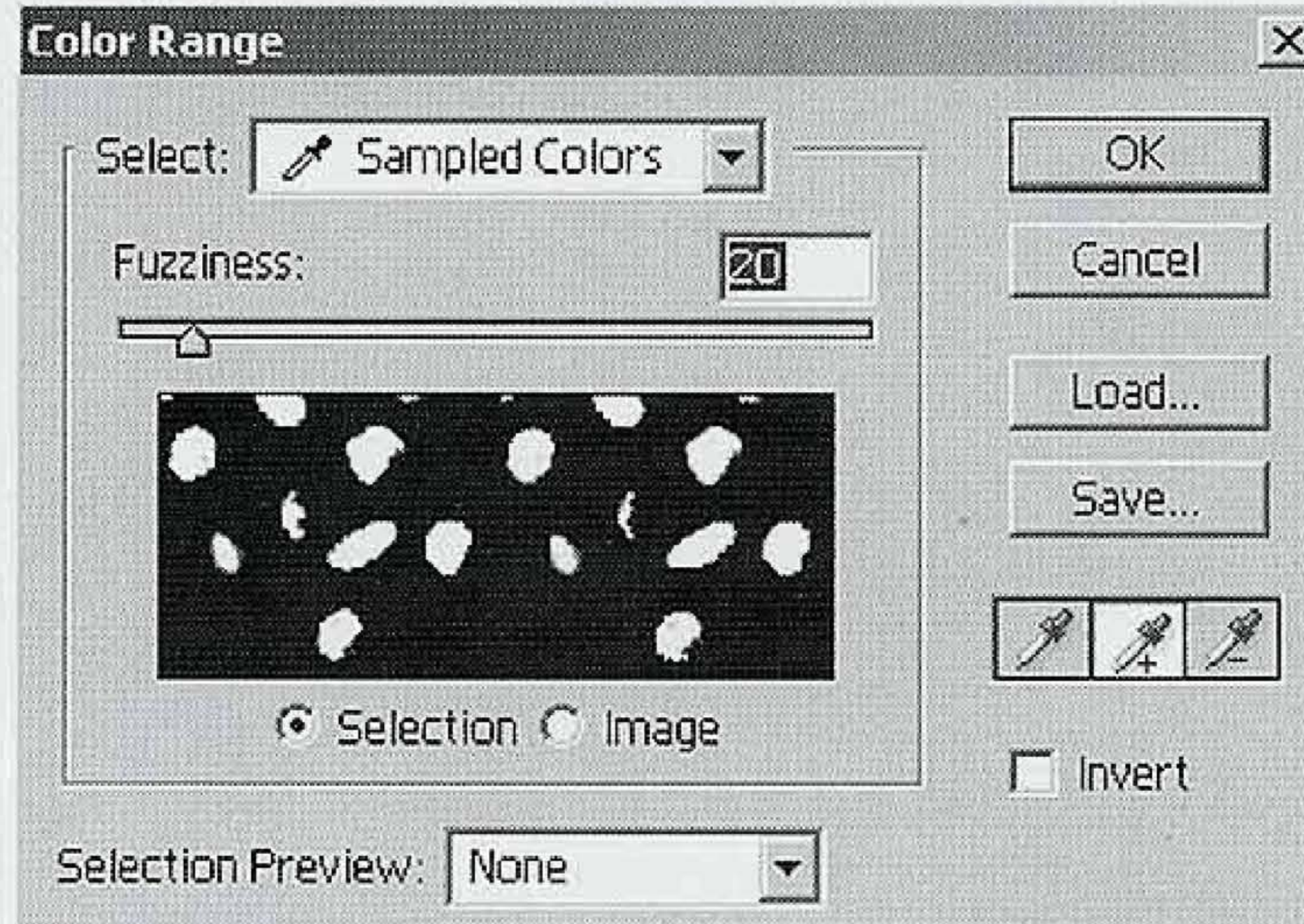

Figure 1: Here is an example of the Color Range dialogue box.

Minimum/Maximum. When more severe blobbing is necessary, use the Minimum/Maximum filters. Maximum "grows" lighter areas, Minimum grows darker areas by clumping pixels into larger squares and "growing" by the value set in the dialogue box in Radius. These are especially useful when clumps of aggregates need to be counted as clumps rather than as individual particles. It can also help to blob odd shapes, such as incomplete circles and two crescents that should have made a donut shape ultimately to be considered one object and not two. Minimum/Maximum can also be used together to eliminate light or dark features, and then to regrow the original, desired features to approximately the same size. This tool may aid in automatic counting more than anything else.

Separate Channels when using Color. RGB Color images can be separated into their red, green or blue color components or channels. The individual channels will show complementary colors as darker and similar colors as lighter. The blue channel, then, will reveal yellows and browns as dark and blue values as light (useful for DAB and peroxidase staining), the red channel will show greens as dark and red values as light, and the green channel will darken reds versus greens. Separate color channels by selecting Show Channels under Window on the menu. Click on the arrowhead at the top, right for the drop down list, then choose Split Channels (the image cannot contain layers or Split Channels will be grayed out).

Use one of these channels if colors separate nicely into dark features of interest and light areas which contain unwanted features. Otherwise choose Merge Channe/s in the Channels dialogue box to remake the original color image (you will be prompted for each channel during the process of merging: simply click okay to each prompt). 


\section{precision \\ powering \\ productivity}

(t:

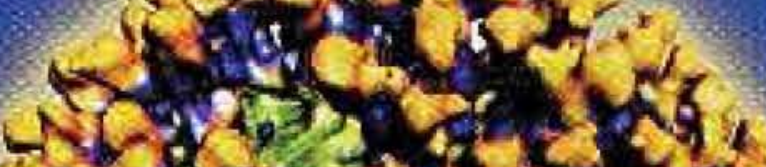

s.t.

2 30

375 s. $^{2}$.

4.95 .

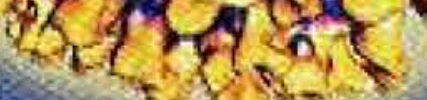

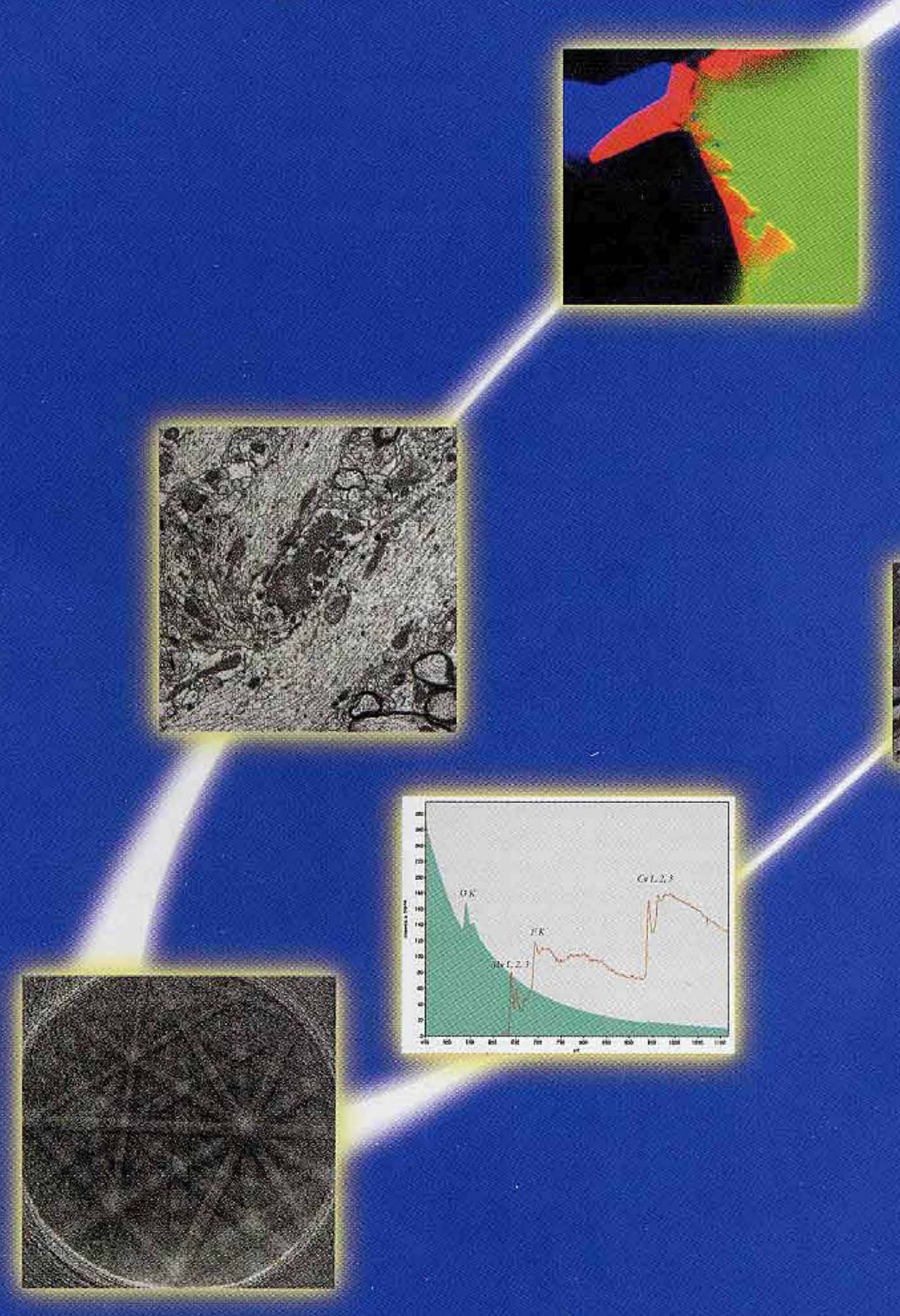

Imaging Systems

Electron energy loss spectrometers

Cathodoluminescence Systems

Cryo-stages

Specimen Preparation

Specimen Holders

8.

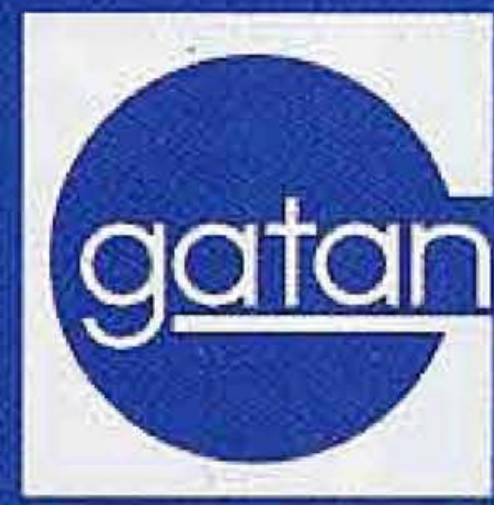

Microscopy Software

a complete solution for TEM and SEM 

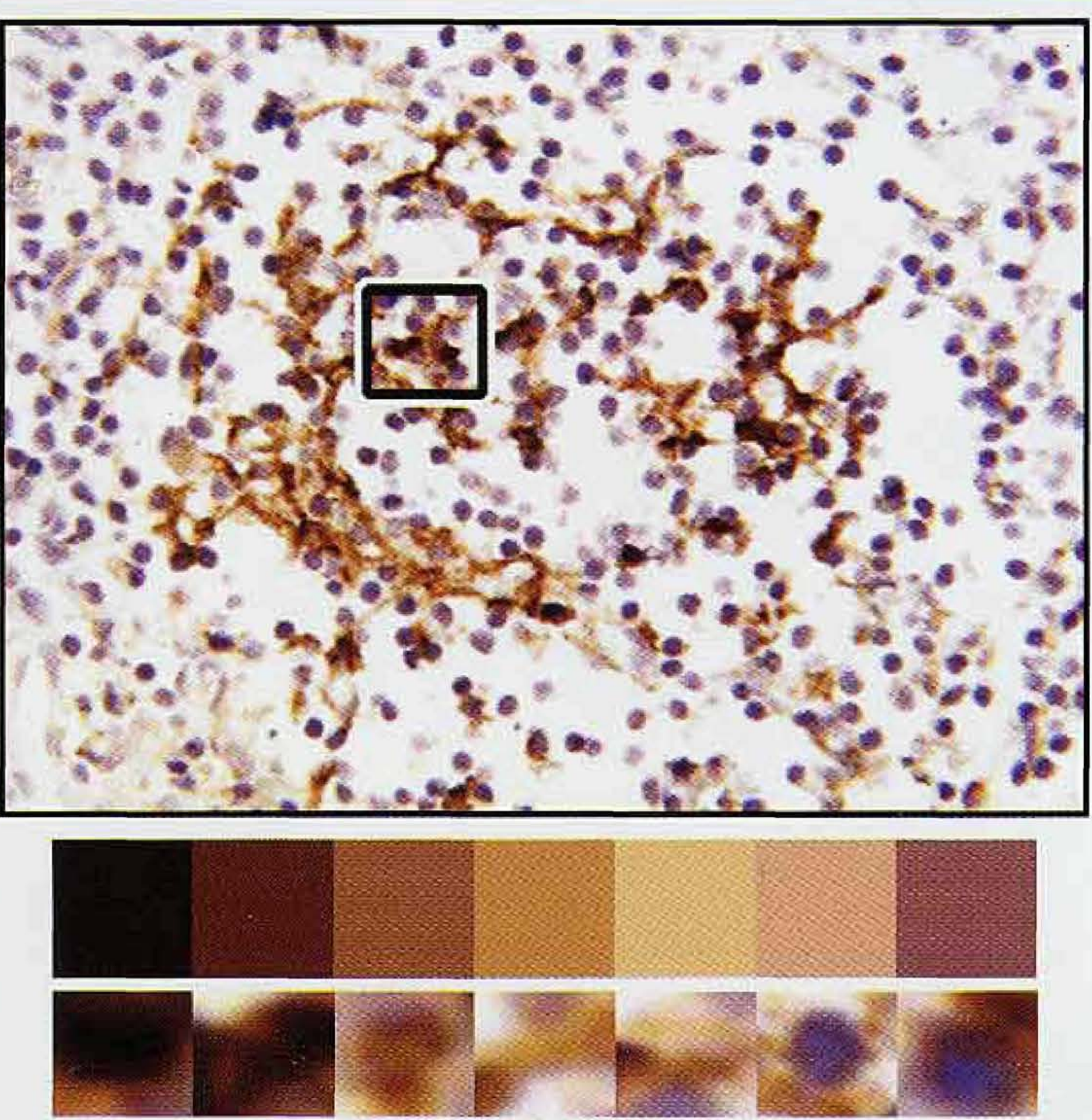

Figure 2: Example of brightfield image stained with $D A B$ and hematoxylin. Brown, DAB staining indicates level of infection. When using Color Range eyedropper tool to click on features in the image, the color squares at the bottom show the many shades called "brown," ranging from black to a saturated mauve. This is shown to demonstrate that many shades make up what our eyes would call a singular color. The areas which contain these shades of brown lie underneath each square of solid color. Note how in some instances we simply do not see the solid square of a particular color in the tissue area beneath. Our eyes interpret the color of interest based upon neighboring colors, making some hues impossible to see by eye, but not by computer. The boxed region is the area used when generating hues, and it is also the area used in Figure \#3 to show the Color Range method.

Selecting Regions of Interest in a Color Channel. Invert the image (Under Image select Adjust or Adjustments and then Invert), then use procedures outlined in the last issue of Microscopy Today to find regions of interest (High Pass Filter, then Threshold). Or, use Color Range to select dark (or light) features using the Color Range procedure.

Color Range Procedure. Whether the image is grayscale or color, the best global segmentation tool is located under Select on the menu, and it is called Color Range. This is the starting point. Any other processing of the image or the selection should be done so that the use of Color Range results in a better ability to select only features of interest. These manipulations include expand/ contract/smooth options for the selection itself to eliminate small or large features (which act like a boolean open/close filters), detailed later. Other manipulations of the image can be done in scientific quantization programs after segmenting in Photoshop. These may include the use of the watershed filter to split apart two cells that adjoin (works best on circular shapes), cutoff filters to eliminate large or small features (when more sophisticated tools are used than what can be done in Photoshop) and "roundness" or "shape factor" filters to eliminate or keep features that are at certain levels of circularity.

Color Range is applied to the whole image unless an area is selected by the user with the lasso or marquee tool. This tool works by selecting the colors or densities you choose by clicking the arrowhead to reveal the Select drop down list. There, you can choose various colors from red to magenta, or three density ranges comprising Highlights, Midtones or Shadows.

Choosing regions of interest by clicking on desired features in the image itself. Most the time, however, you identify which colors or densities you want Color Range to find. Before getting started, double click on the Eyedropper Tool in the toolbar and choose a " $3 \times 3$ Average" sample size (on the menu in $6 x, 7 x$ and in the dialogue box in $4 x, 5 x$ ). In the Color Range dialogue box, choose Sampled Colors next to Select. You will want to choose the first color or density on your image by using the eyedropper tool to click on a feature of interest. Then you will need to choose the plus (+) eyedropper tool to click on more hues or densities. Choose several features of interest: 10 or so locations. The slider under Fuzziness then expands or contracts the range of colors based upon the color and density values you have clicked on in the image itself.

So, for example, if you have clicked first on a mid-red with pixel values of 0 green, 0 blue and 128 red, the slider can be increased to include values of red on either side of 128 (a range) depending on the position of the slider (the mathematical calculation of the relationship of that slider to "range," is, unfortunately, proprietary: Adobe

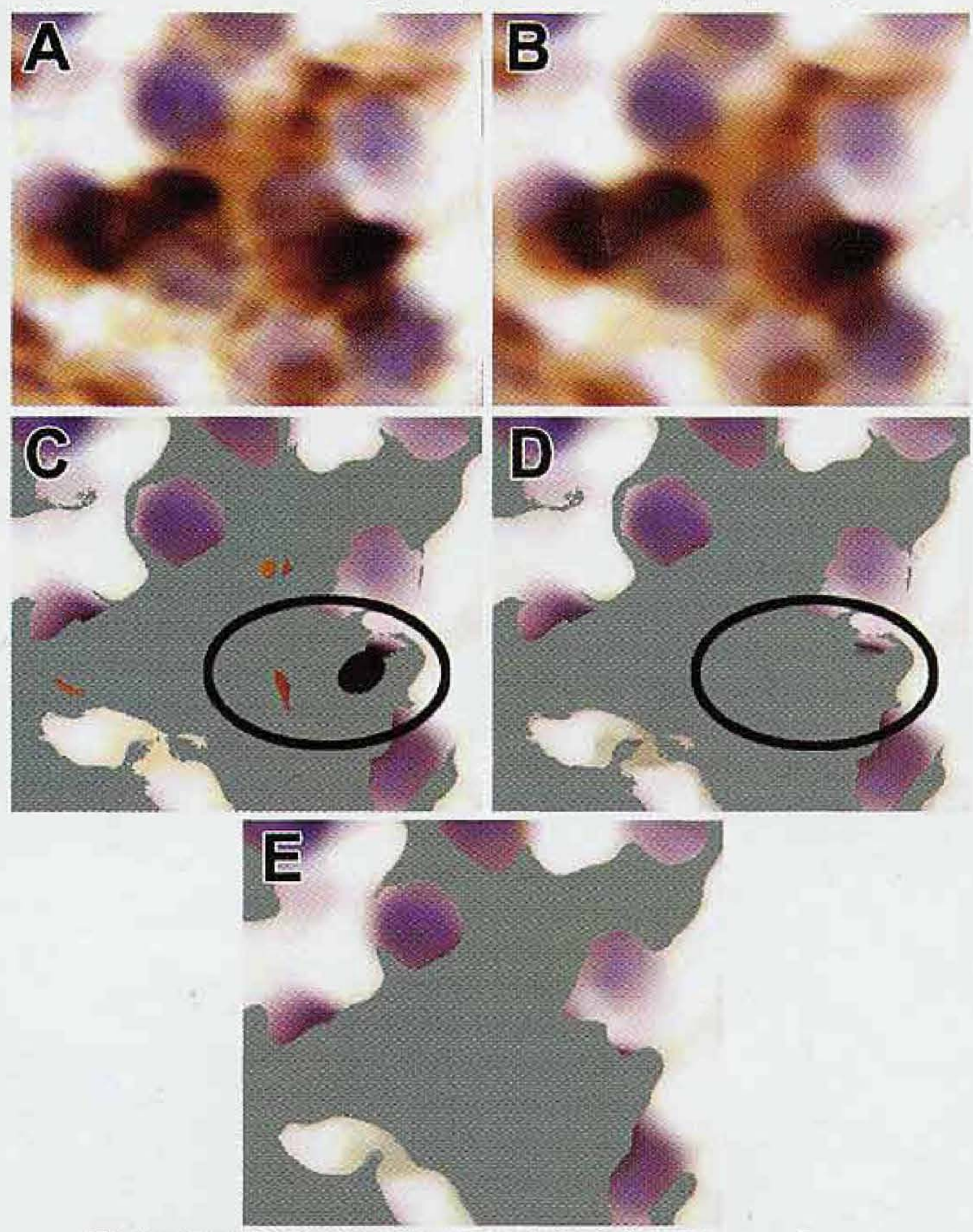

Figure 3: Panel A shows the original, "raw" image from boxed area of Figure \#2. Panel B shows the same area after the application of a median filter (radius set to 8 ). The greenish overlay on Panel C shows the amount of DAB-stained tissue selected by Color Range after the first attempt. Note the inability for the Color Range filter to include reddish brown and black, even though these colors may have been clicked on. Panel D shows the inclusion of reddish-brown and black with the addition of 2 more steps in which Color Range was applied. Panel E shows the area chosen by the greenish overlay after adding all the selections together from 3 separate Color Range files, and then smoothed with a value of 4 to eliminate fine selections. This selection can be modified further by using Expand or Contract if the area is thought to be incorrect. 
-

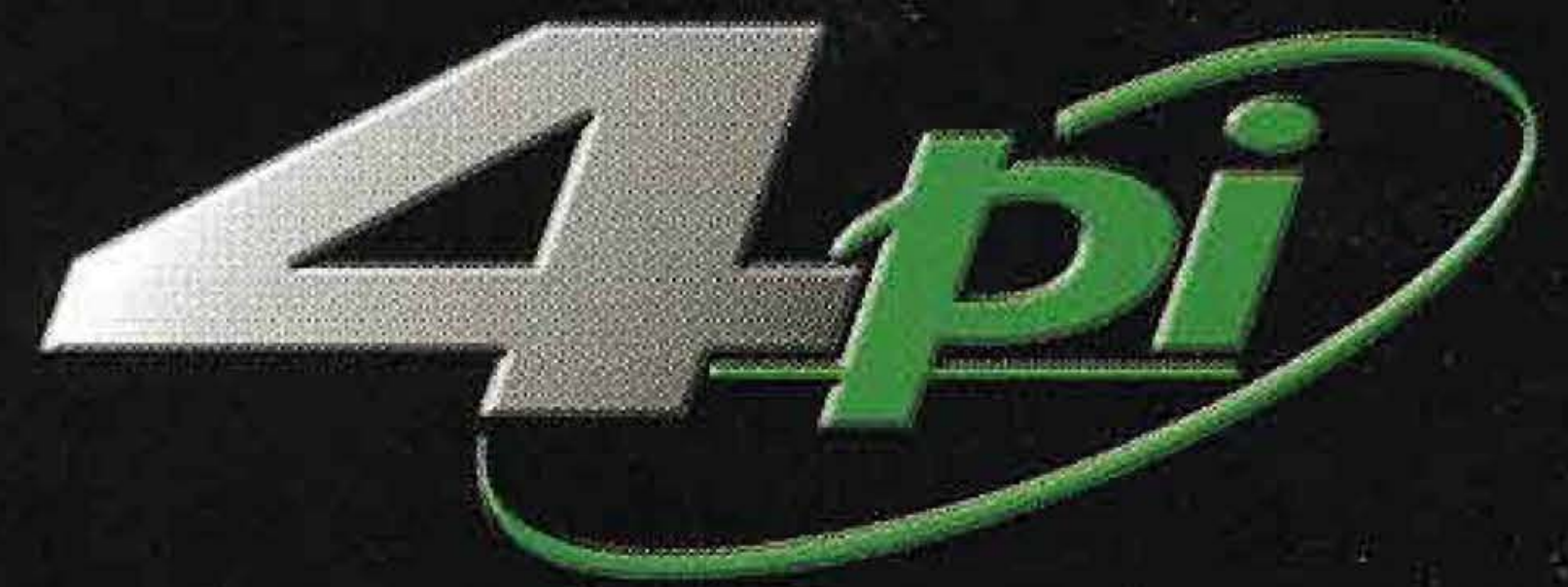

EDX \& Imaging Systems

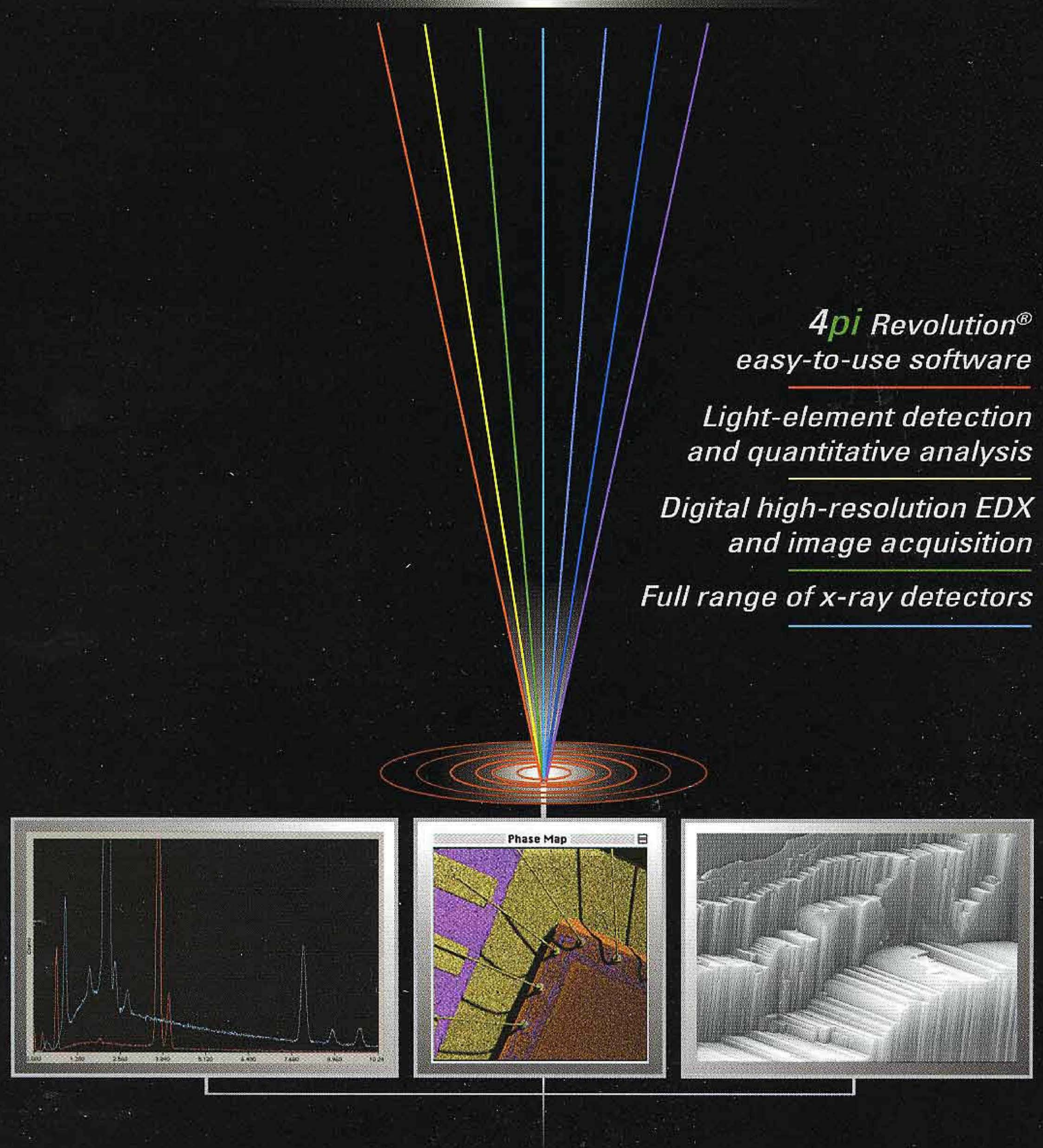




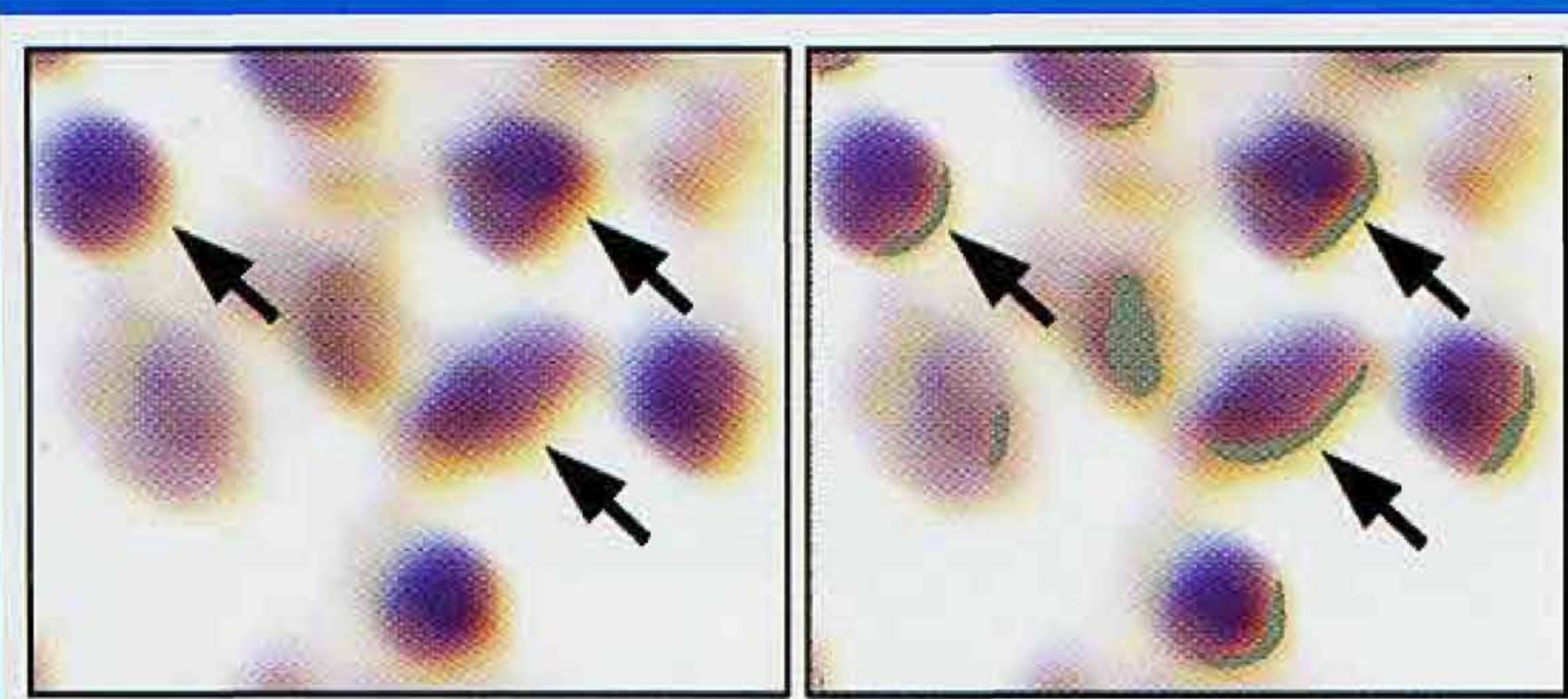

Figure 4: The individual cells contain brown at one side, probably a result of diffraction when acquiring the image. Because of that, quantization from this sample would include noninfected areas along with infected areas. This sample must be reacquired under more ideal conditions, or manual outlining methods need to be employed (perhaps "speeded-up" by the use of the Wand tool).

Corporation will not reveal that information). If you have clicked on Selection (which exists under the preview image within the dialogue box) and the Selection Preview is set to None, you will see white features suggesting what will be selected, and black to suggest what will not be selected. The word "suggest" is purposeful: the visual image does not always translate into what Color Range chooses, and the Fuzziness setting may take a few tries before you are satisfied.

In any case, it is best to keep the Fuzziness at a low setting (1 - 25: the greater the range and variety of hues within the same color, the lower the value) while clicking with the plus eyedropper tool on many different shades of the color of interest and density range. In that way, the selection becomes far more defined and the range narrowed. Do not be too aggressive: most biological images contain a wide range of the color of interest, and not always closely related. In the figure accompanying this article, the hue thought of as "brown" ranges from black to red-brown to yellow-brown to mauve. We have the intrinsic ability to see brown, for example, when it fringes a black center and we are likely to call that feature "brown," (when it is hardly brown at all). We want the computer to also see brown in that manner, but it sees the black center in these features and that density of black everywhere else as well, especially when the Fuzziness setting is too high.

Because of that, many images require several steps in which Color Range is used for more and more refined selections, and this article will assume a "worst case" image. You may, however, be fortunate enough to use Color Range only once in order to select all your regions of interest if the staining is done well, the tissue is right, and the stars in heaven are aligned.

In any case, you MUST save your Color Range choices (.AXT file) by clicking Save in the Color Range dialogue box. These hues and densities will then be chosen in subsequent images. Test a few of these to be sure your saved selection works equally well on other images. Remember that it may be best to eliminate unwanted regions manually in subsequent images with the lasso tool rather than hope to automatically select with the saved Color Range values for subsequent images.

Most will find that the first saved Color Range choices (.AXT file) are only a starting point. This may select most of the colors/densities of interest, but not all. If that is the case, then the excluded colors/densities need to be marked. I find the easiest method for marking to be the eyedropper tool on the toolbar. If the Shift key is held down as you click with the eyedropper tool on the image, a crosshair (of sorts) is left be- hind (note that the crosshair disappears when another tool is selected on the toolbar, and that the crosshair is not part of the image). You can mark up to 4 locations in this manner. If it is difficult to see what has been selected, click on the Quick Mask Mode icon at the bottom of the toolbar (or simply type the letter $\mathrm{Q}$ : type again to eliminate mask). What is not selected will be covered in a red overlay.

Once you have marked these locations, save your "marching ants" selection if this is to be more than one go-around. Under Select on the menu choose Save Selection. In the Save Selection dialogue box, give the selection a name. Make sure you deselect (under Select choose Deselect or use control/ command + D keys), or Color Range will only work on areas defined by your "marching ants" selection.

Now return to Color Range and open its dialogue box. This time click on one marked location, increase Fuzziness until that location - and others in the same color/density range-are white on the preview, then be sure to click Save and give it a name. Click Okay in the dialogue box and the "marching ants" selection will show on the screen. That selection will also have to be saved. Under Select on the menu again choose Save Selection. In the Save Selection dialogue box, give the selection a different name. Repeat this process again until all regions of interest are selected.

Once you are satisfied that all regions of interest have been selected through several saved Color Range files, then add all the saved selections together. Under Select, choose Load Selection. Open the first selection, then, open subsequent selections. When additional selections are opened, be sure to click Add to Selection in the Load Selection dialogue box. Now you should have all color values/densities within your regions of interest selected.

Contract/Expand/Smooth/Border. Finally, the added-together selection might still be too large, too small, it may contain structures that are too detailed, or the desired region of interest may exist in an orbit (donut shape) around the objects that are selected. All of these can be changed by choosing any one of these functions under Select and then Modify. Note that Contract and Expand can be used together to eliminate tiny selections (first use Contract at a value of 1 or so to deselect small objects, then use Expand at the same value). Smooth is used to ignore finely detailed edges. And Border is used for making an orbit around objects. The orbits mimic the shape of the object, and these can be added so that several larger and larger orbits can be made.

Once all these steps have been tested on subsequent images of the same kind, then the entire series of steps should be saved to an action. In that manner, one press of a button will apply all these steps to all your images. Be sure to include the concluding steps in your action: The final selection will then need to be filled with black (Under Edit, choose Fill then select Black), then invert the selection (Select/Invert) to fill with white. Be sure to eliminate any alpha planes in channels-what was created when selections were saved. Under Window select Show Channels or Channels (if not already visible on the screen). Click and drag each alpha channel to the trash can at the bottom of this dialogue box. Then save the image for subsequent quantization in your favorite program.

N.B.: Photoshop and Adobe are trademarks of the Adobe Corporation. 


\section{IXRF Expands "Coast to Coast" celebrating their 10 year anniversary}

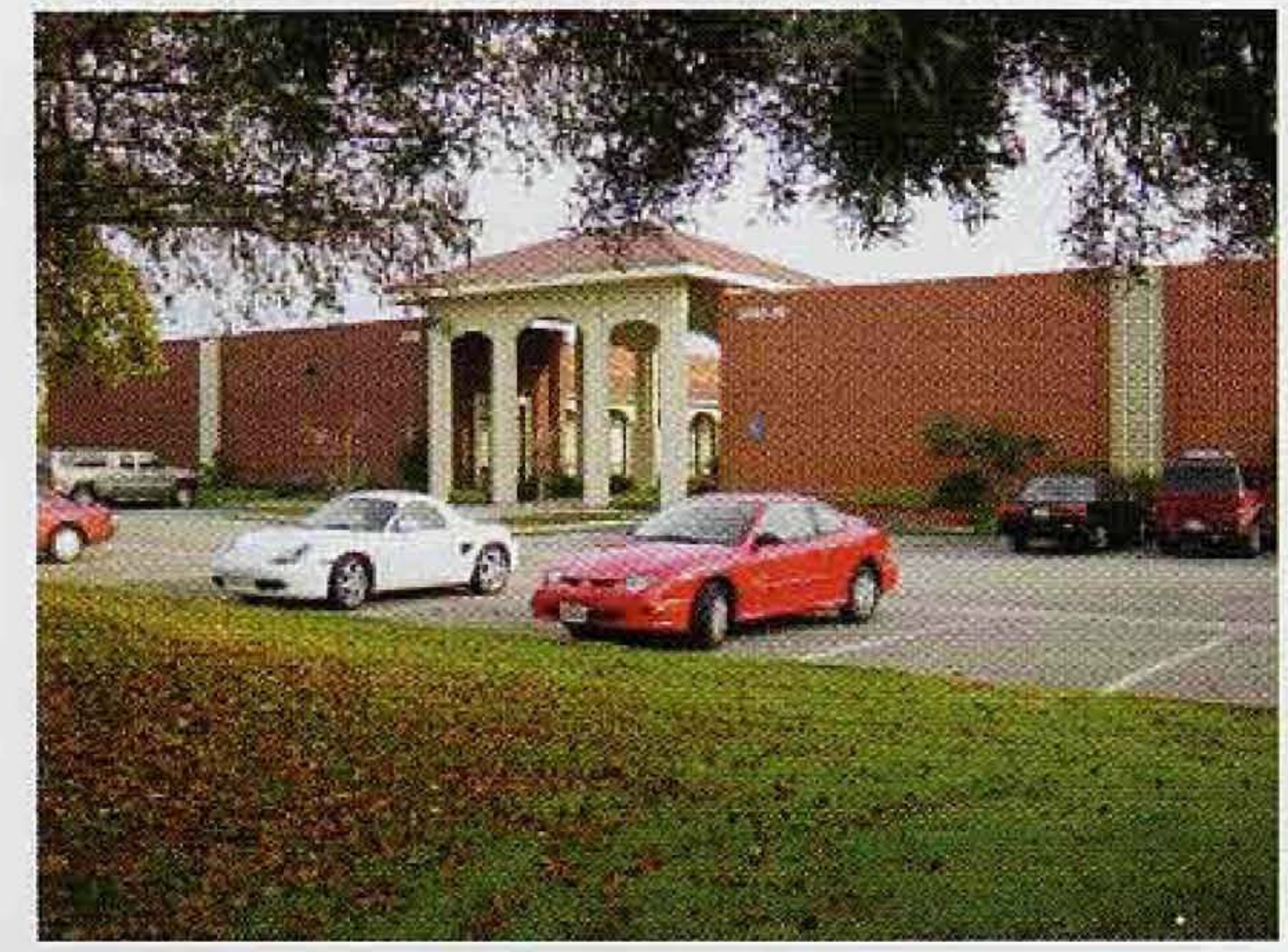

IXRF's Silicon Valley development facility

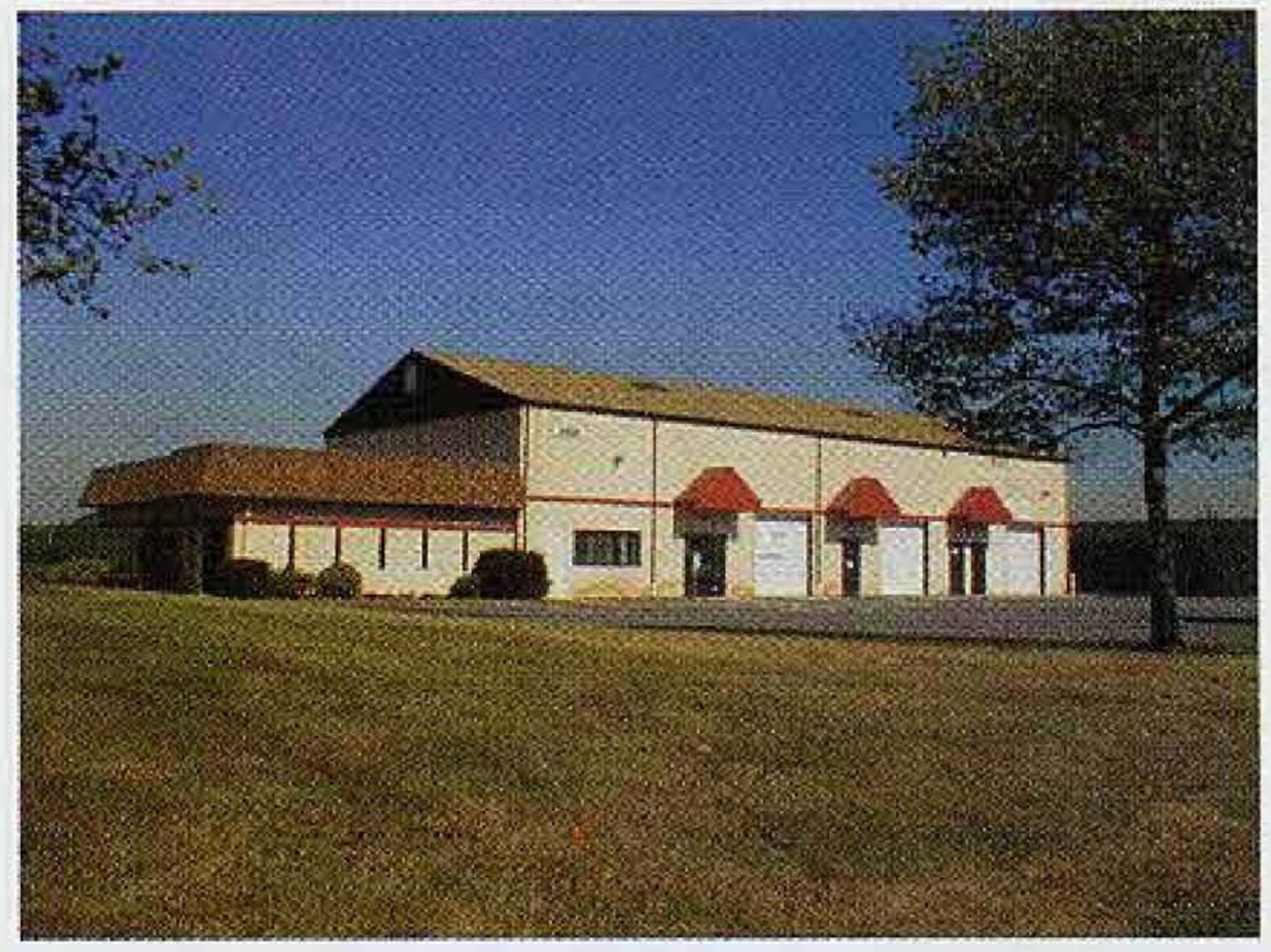

IXRF's Trenton New Jersey facility

IXRF thanks its loyal customers for their support in the growth of IXRF. At IXRF, we believe "satisfied" customers is the best way to sell more systems. By offering free software upgrades, customers never have to purchase another system. Even IXRF's first customers, 10 years ago, can still download the latest software and have all the latest features. Through customer suggestions, IXRF's software has become the leading Microanalysis software rivaling the major EDS companies - at a fraction of the cost. To our customers, thanks again!

\section{Major Milestones}

1992: IXRF personnel begin planning future products.

1993: Los Alamos National Lab receives the first IXRF system.

1995: Digital Imaging, Feature analysis, and X-Ray mapping are added into the systems.

1997: IXRF completes the first $100 \%$ Integrated EDS Microanalysis system in history (developed for LEO Electron Microscopy).

1998: Jetscan Engine Health Monitor wins a millenium award from the British government (developed for LEO Electron Microscopy).

1999: IXRF Completes the "Particle Scan" offering the first truly integrated Particle Analysis inside the operating system of the SEM (developed for LEO Electron Microscopy).

1999: IXRF designs a new hardware interface allowing the use of Oxford PentaFET detectors for upgrading existing customers.

2000: IXRF completes the second fully Integrated EDS system, this time for JEOL Ltd.

2001: IXRF completes Particle Analysis on the Integrated JEOL Ltd product line.

2002: IXRF mounts the first micro $x$-ray tube on an SEM, to offer the first fully-integrated XRF and EDS microanalysis within the SEM.

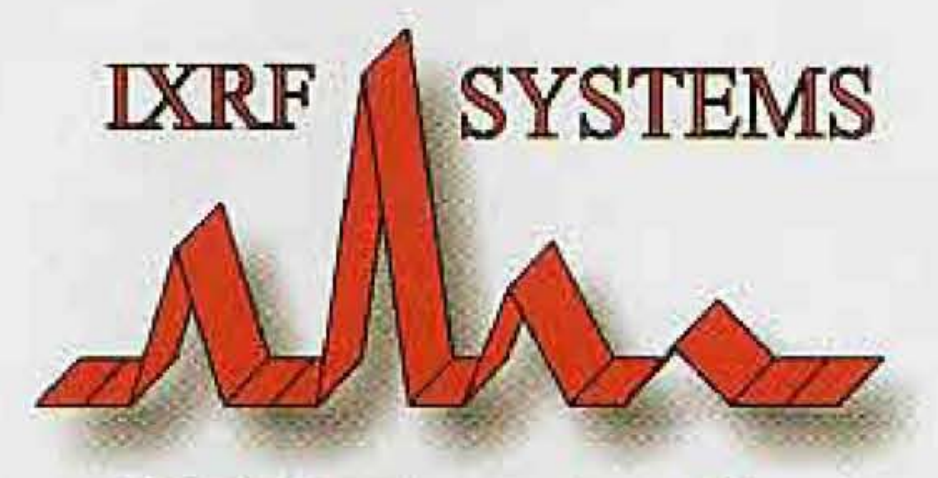

IXRF Systems, Inc., Houston, TX, USA, www. ixrfsystems.com $\mathrm{Ph}:(281)$ 286-6485 Fax:(281)286-2660

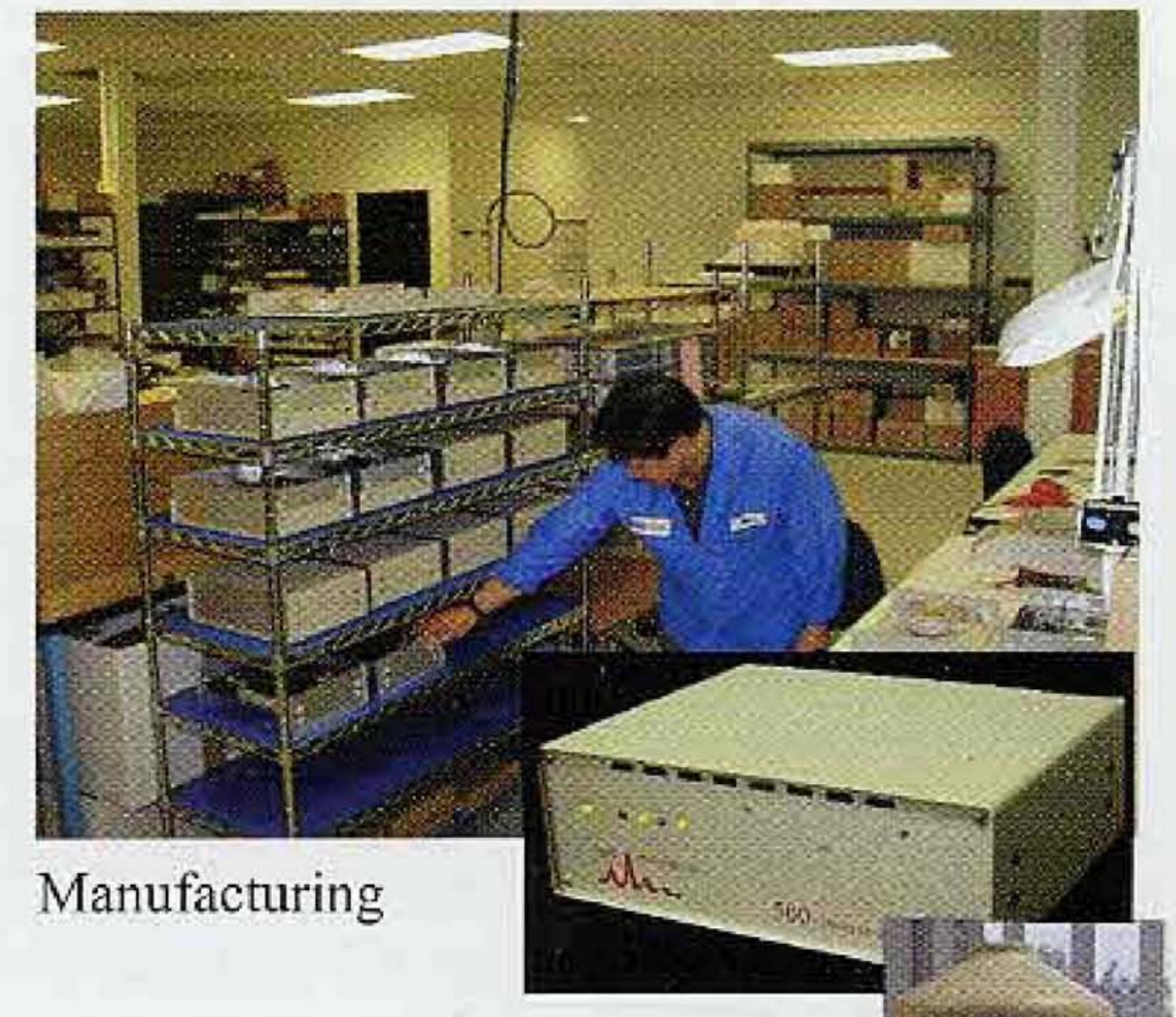

New Detectors

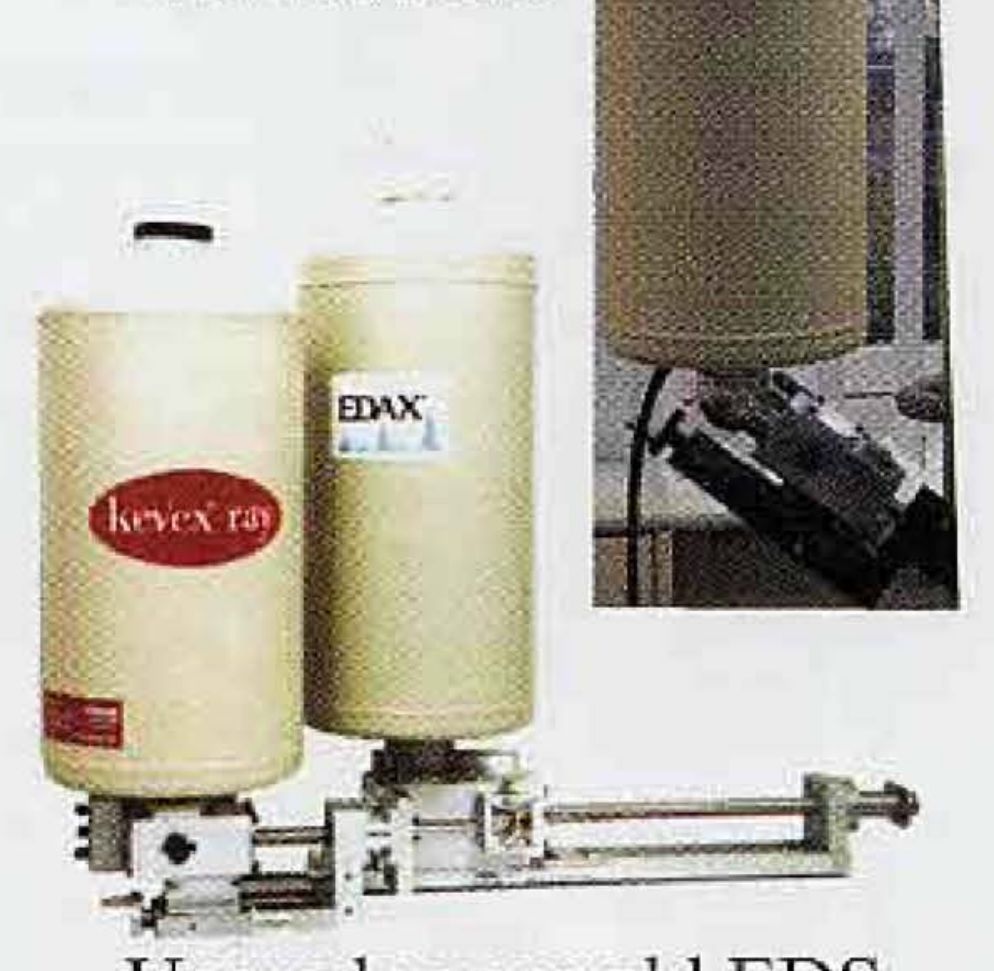

Upgrade your old EDS analyzer with a PC, but keep the detector! 